Pacific Journal of Mathematics

CLOSED RANGED RESTRICTION OPERATORS ON 


\section{CLOSED RANGED RESTRICTION OPERATORS ON WEIGHTED BERGMAN SPACES}

\section{DANIEL LUECKING}

We extend the results in [5] to several variables and to larger classes of domains. In particular it is shown that if $B$ is the unit ball in $C^{n}$ and $G \subseteq B$ is measurable then for any $p<0$

$$
\int_{B}|f|^{p} d V \leq \text { const. } \int_{G}|f|^{p} d V,
$$

for all analytic functions $f$ in $L^{p}(B)$ if and only if there exist $\delta>0$ and $0<r<1$ such that $|G \cap Q(B(0, r))| \geq \delta|Q(B(0, r))|$ for all automorphisms $Q: B \rightarrow B$. This is actually done for weighted integrals in more general domains. This is easily seen to be a criterion for the operation $\left.f \rightarrow f\right|_{G}$ to have closed range. In addition, some partial results on the closed range of $f \rightarrow\left\{f\left(z_{n}\right)\right\}$ in some weighted $l^{p}$ spaces are obtained for sequences $\left\{z_{n}\right\}$ in certain domains.

1. The question to be investigated here can be formulated as follows: Let $\Omega$ be an open set in $\mathbf{C}^{n}$ and let $G$ be a measurable subset of $\Omega$. Let $A$ be some space of analytic functions on $\Omega$ and $E$ some space of functions on $G$ such that $\left.f\right|_{G} \in E$ for all $f \in A$. The question: when will $\left\{\left.f\right|_{G}: f \in A\right\}$ be a closed subspace of $E$ ? Here the investigation will be limited to $L^{p}$ norms.

Let $\Omega$ be as above and let $w$ be a non-negative measurable function on $\Omega$. Let $d V$ denote the $2 n$-dimensional volume measure on $\mathbf{C}^{n}$. For $p>0$, define

$$
A^{p}(w):=\left\{f: f \text { is holomorphic on } \Omega \text { and } \int_{\Omega}|f|^{p} w d V<+\infty\right\}
$$

and

$$
\|f\|_{p, w}:=\left(\int_{\Omega}|f|^{p} w d V\right)^{1 / p}
$$

If $p \geq 1$, then $\|\cdot\|_{p, w}$ is a norm, otherwise $\|\cdot\|_{p, w}^{p}$ is a metric. I will assume $w$ is continuous and non-vanishing in $\Omega$. In this case $A^{p}(w)$ is a Banach space when $p \geq 1$ and is otherwise a complete topological vector space. 
In case $p=2$ and $w \equiv 1, A^{2}:=A^{2}(1)$ is a Hilbert space with a reproducing kernel. That is, there is a function $B_{\Omega}(z, \zeta)$ on $\Omega \times \Omega$ such that

$$
f(z)=\int_{\Omega} B_{\Omega}(z, \zeta) f(\zeta) d V(\zeta), \quad f \in A^{2} .
$$

The function $B_{\Omega}$ is called the Bergman kernel. The subscript will be omitted when clarity will not suffer so $B_{\Omega}$ becomes $B$. So that certain results are not vacuous, it will always be assumed that $\Omega$ is such that $A^{2}$ contains sufficiently many functions. Specifically, I shall work in the setting set forth in Coifman and Rochberg [3]. Actually, I shall only need three properties of $\Omega$ beyond the existence of non-trivial elements in $A^{2}$. The first of these is that $\Omega$ is homogeneous. This means the automorphism group of $\Omega$ is transitive, i.e. that for any $z_{1}, z_{2} \in \Omega$ there is a biholomorphic map $Q$ of $\Omega$ onto $\Omega$ such that $Q\left(z_{1}\right)=z_{2}$. The other two properties of $\Omega$ that are needed require the introduction of the Bergman distance function $d(z, \zeta)$. This distance is invariant under biholomorphic automorphisms of $\Omega$ and on compact subsets of $\Omega$ it is equivalent to the Euclidean distance. The definition of $d$ and $B$ and further properties can be found in books by Bailey [1] and Kobayashi [4].

The second property $\Omega$ is to have is that $d\left(z_{0}, \zeta\right) \rightarrow+\infty$ as $\zeta$ tends to any boundary point of $\Omega$ (including $\infty$ ), where $z_{0} \in \Omega$. Note that this property is preserved under biholomorphic maps because the Bergman distance is preserved. The third property needed is that there exist positive constants $\eta$ and $C$ such that

$$
\frac{B(z, z)}{B(w, w)}<C \quad \text { whenever } d(z, w)<\eta .
$$

Notice that if there exists such a $C$ for some $\eta$ then there exists one for any $\eta$.

All of these properties are satisfied by balls and half-planes. The last property is satisfied by a class of domains called "symmetric Siegel domains of type two". This is an easy consequence of Lemma 2.3 in [3].

The major result of this paper is the following sufficient condition for the closure of the range of a restriction operator on certain weighted Bergman spaces. Here $|\cdot|$ denotes $2 n$-dimensional volume and $S(a, r)=$ $\{z \in \Omega: d(a, z)<r\}$.

THEOREM 1. Let $\Omega \subseteq C^{n}$ be a domain with the following properties

(i) $\Omega$ is homogeneous

(ii) For any $z \in \Omega, d(z, \zeta) \rightarrow+\infty \zeta \rightarrow \partial \Omega$. 
(iii) There exist $\eta, C>0$ such that $B(z, z)<C B(\zeta, \zeta)$ whenever $d(z, \zeta)<\eta$.

Let $w(z):=B(z, z)^{\alpha}$ where $\alpha \in R$ is chosen so that $A^{p}(w)$ is non-trivial. Suppose $G$ is a measurable subset of $\Omega$ and $r>0$ such that

$\left(L_{r}\right) \quad$ For some $\delta>0,|G \cap S(a, r)|>\delta|S(a, r)|, \quad a \in \Omega$,

then

$$
\int_{\Omega}|f|^{p} w d V \leq K \int_{G}|f|^{p} w d V, \quad \text { all } f \in A^{p}(w),
$$

where $K$ is a constant which does not depend on $f$.

As a consequence the restriction map $R: A^{p}(w) \rightarrow L^{p}(G, w)$ has closed range.

This result was proved in [5] for the case where $D$ is the unit disk in $\mathbf{C}$. Two of the lemmas needed for that proof go through for the present case with little change. The third requires technical adjustments but its spirit is the same. The proof of the above theorem is the subject of $\$ 2$, where a converse will also be presented under additional assumptions.

Section 3 will deal with sufficient conditions that the restriction of functions in $A^{p}(w)$ to a sequence in $\Omega$ defines an operator with closed range in a certain sequence space. This will use techniques from $\S 2$. Other restriction operators will be briefly considered.

In $\S 4$, the case $p=\infty$ will be discussed for comparison. In addition an example will be presented which shows that the phrase " $f \in A^{p}(w)$ " in (1) cannot be omitted. That is, there is an example of a function, $f$, holomorphic in $\Omega$ such that $\int_{\Omega}|f|^{p} w d V=+\infty$ but $\int_{G}|f|^{p} w d V<+\infty$ for a certain $G$ satisfying the hypotheses of the theorem. Thus the finiteness of the right-hand side of (1) does not imply the left-hand side is finite, but if it $i$ finite then the inequality is valid.

2. The proof takes three lemmas, the first of which does not depend on the analyticity of the functions in question and is used only in the proof of the second and in $\S 3$. Constants are denoted by $C$ which may indicate a different constant from one occurrence to the next. Subscripts serve merely to indicate the difference between nearby occurrences and, for example, $C_{1}$ in Lemma 1 need not equal $C_{1}$ in Lemma 2.

LEMMA 1. Let

$$
A_{\varepsilon}=\left\{a \in \Omega:|f(a)|^{p} \leq \varepsilon\left(\int_{S(a, r)}|f|^{p} d V\right) /|S(a, r)|\right\}
$$


and suppose $\int|f|^{p} w d V<+\infty$. Then there is a constant $C>0$ not depending on $f$ such that

$$
\int_{A_{\varepsilon}}|f|^{p} w d V<C \varepsilon \int_{\Omega}|f|^{p} w d V
$$

Proof. For $a \in A_{\varepsilon}$

$$
|f(a)|^{p} \leq \varepsilon \frac{1}{|S(a, r)|} \int_{\Omega} X_{S(a, r)}(z)|f(z)|^{p} d V(z) .
$$

Multiply by $w(a)$ and integrate over $A_{\varepsilon}$. Then apply Fubini's theorem to the right-hand side. This gives

$$
\begin{aligned}
& \int|f(a)|^{p} w(z) d V(a) \\
& \quad \leq \varepsilon \int_{\Omega}|f(z)|^{p}\left[\int_{A_{\varepsilon}} \frac{1}{|S(a, r)|} X_{S(a, r)}(z) w(a) d V(a)\right] d V(z) .
\end{aligned}
$$

Observe that $X_{S(a, r)}(z)=X_{S(z, r)}(a)$ and that $w(a)<C w(z)$ for some $C>0$ provided $a \in S(z, r)$. This yields

$$
\begin{aligned}
& \int|f(a)|^{p} w(a) d V(a) \\
& \leq C \varepsilon \int_{\Omega}|f(z)|^{p} w(z)\left[\int_{S(z, r)} \frac{1}{|S(a, r)|} d V(a)\right] d V(z) .
\end{aligned}
$$

It must be shown that the expression in brackets is bounded independent of $z$.

Let $b \in \Omega$ be fixed. The invariance property of the Bergman kernel gives

$$
B(Q(z), Q(z))\left|\operatorname{det} Q^{\prime}(z)\right|^{2}=B(z, z)
$$

where $Q$ is any automorphism of $\Omega$. Now,

$$
\begin{aligned}
\int_{S(a, r)} B(z, z) d V(z) & \\
& =\int_{S(b, r)} B(Q(z), Q(z))\left|\operatorname{det} Q^{\prime}(z)\right|^{2} d V(z)
\end{aligned}
$$


where $Q$ is an automorphism of $\Omega$ mapping $b$ to $a$. Thus, if $C_{1}$ is the constant in hypothesis (iii),

$$
\begin{aligned}
\int_{S(a, r)} d V(z) & \leq \frac{C_{1}}{B(a, a)} \int_{S(a, r)} B(z, z) d V(z) \\
& =\frac{C_{1}}{B(a, a)} \int_{S(b, r)} B(z, z) d V(z)=\frac{C_{2}}{B(a, a)}
\end{aligned}
$$

So $|S(a, r)|<C_{2} / B(a, a)$. Similarly, there is a constant $C_{3}$ so that $|S(a, r)|>C_{3} / B(a, a)$. Thus

$$
\begin{aligned}
\int_{S(z, r)} \frac{1}{|S(a, r)|} d V(a) & \leq \frac{1}{C_{3}} \int_{S(z, r)} B(a, a) d V(a) \\
& \leq \frac{C_{1}}{C_{3}} B(z, z)|S(z, r)| \leq \frac{C_{1} C_{2}}{C_{3}} .
\end{aligned}
$$

Now let $0<\lambda<(1 / 2)^{p}$ and define, for $f \in A^{p}(w), E_{\lambda}(a):=$ $E_{\lambda}(f, a):=\{z \in S(a, r):|f(z)|>\lambda|f(a)|\}$. Let $B_{\lambda} f$ be defined by

$$
B_{\lambda} f(a)=\frac{1}{\left|E_{\lambda}(a)\right|} \int_{E_{\lambda}(a)}|f|^{p} d V .
$$

LEMMA 2. Let $f \in A^{p}(w)$ and $B_{\varepsilon}=\left\{a \in \Omega:|f(a)|^{p}<\varepsilon^{1+2 n / p} B_{\lambda} f(a)\right\}$. Then

$$
\int_{B_{\varepsilon}}|f|^{p} w d V<C \varepsilon \int_{\Omega}|f|^{p} w d V
$$

where $C$ is independent of $f$.

Proof. Let $A_{\varepsilon}$ be as in Lemma 1. Then

$$
\int_{B_{\varepsilon}}|f|^{p} w d V \leq \int_{A_{\varepsilon}}|f|^{p} w d V+\int_{B_{\varepsilon} \backslash A_{\varepsilon}}|f|^{p} w d V
$$

so it suffices to prove the lemma with $B \backslash A$ replacing $B$. Proceeding as in Lemma 1 we obtain

$$
\begin{aligned}
& \int_{B_{\varepsilon} \backslash A_{\varepsilon}}|f|^{p} w d V \\
& \quad<C_{1} \varepsilon^{1+2 n / p} \int_{\Omega}|f(z)|^{p} w(z)\left[\int_{B_{\varepsilon} \backslash A_{\varepsilon}} \frac{X_{E_{\lambda}(a)}(z)}{\left|E_{\lambda}(z)\right|} d V(a)\right] d V(z) .
\end{aligned}
$$


It remains to show that the integral in brackets is bounded by $C_{2} / \varepsilon^{2 n / p}$ where $C_{2}$ is independent of $z$. To do this, observe that $X_{E_{\lambda}(a)}(z) \leq$ $X_{S(a, r)}(z)=X_{S(z, r)}(a)$, and thus the bound will be established if it can be shown that $\left|E_{\lambda}(a)\right|>\left(\varepsilon^{2 n / p} / C_{3}\right)|S(a, r)|$, for then the estimate in Lemma 1 can be used.

Let $b$ again be a fixed point in $\Omega$ (the same one as in Lemma 1) and observe that

$$
z \in E_{\lambda}(f, a) \Rightarrow Q^{-1}(z) \in E_{\lambda}\left(f \circ Q, Q^{-1}(a)\right) .
$$

Let $Q$ be an automorphism of $\Omega$ taking $b$ to $a$. Then

$$
\begin{aligned}
\left|E_{\lambda}(f, a)\right| & =\left|Q\left(E_{\lambda}(f \circ Q, b)\right)\right|=\int_{Q\left(E_{\lambda}(f \circ Q, b)\right)} d V \\
& \geq \frac{C_{4}}{B(a, a)} \int_{Q\left(E_{\lambda}(b)\right)} B(z, z) d V(z) \\
& \geq C_{5}|S(a, r)| \int_{E_{\lambda}(b)} B(z, z) d V(z) . \\
& \geq C_{6}|S(a, r)| \cdot\left|E_{\lambda}(b)\right|
\end{aligned}
$$

where $E_{\lambda}(b)=E_{\lambda}(f \circ Q, b)$. If $a \notin A_{\varepsilon}$, we have

$$
\begin{aligned}
& |f(a)|^{p}>\varepsilon \frac{1}{|S(a, r)|} \int_{S(a, r)}|f|^{p} d V>\varepsilon C_{7} B(a, a) \int_{S(a, r)}|f|^{p} d V \\
& \quad>\varepsilon C_{8} \int_{S(a, r)}|f(z)|^{p} B(z, z) d V(z) \\
& \quad=\varepsilon C_{8} \int_{S(b, r)}|f(Q(z))|^{p} B(z, z) d V(z) \geq \varepsilon C_{9} \int_{S(b, r)}|f(Q(z))|^{p} d V(z) .
\end{aligned}
$$

Thus, with $g=f \circ Q$

$$
|g(b)|^{p}>\varepsilon C_{9} \int_{S(b, r)}|g|^{p} d V .
$$

Without loss of generality, we suppose the integral on the right equals one. Let $K$ be a compact Euclidean ball in $S(b, r)$ centered at $B$. Then $|\operatorname{grad} g|<C_{10}$, where $C_{10}$ depends only on $K$ and $S(b, r)$. Thus

$$
|g(z)-g(b)|<C_{10}|z-b|<\frac{\left(\varepsilon C_{9}\right)^{1 / p}}{2}
$$


provided $|z-b|<\left(\varepsilon C_{9}\right)^{1 / p} / 2 C_{10}$. On the ball about $b$ of radius $\left(\varepsilon C_{9}\right)^{1 / p} /\left(2 C_{10}\right)$ we have

$$
\begin{aligned}
|g(z)| & >|g(b)|-|g(z)-g(b)|>|g(b)|-\frac{\left(\varepsilon C_{9}\right)^{1 / p}}{2} \\
& >\frac{1}{2}|g(b)|>\lambda^{1 / p}|g(b)| .
\end{aligned}
$$

Thus, the ball of radius $\varepsilon^{1 / p} C_{11}$ is contained in $E_{\lambda}(b)$, and so $\left|E_{\lambda}(b)\right|>$ $C_{12} \varepsilon^{2 n / p}$. Combining this with (2) yields the required inequality.

LEMma 3. There is a constant $C$ such that if $f$ is holomorphic in $\Omega, a \in \Omega$ and $\lambda<1$ then

$$
1-\frac{\left|E_{\lambda}(f, a)\right|}{|S(a, r)|}<C \frac{\log \left(C B_{\lambda} f(a) /|f(a)|^{p}\right)}{\log \left(C B_{\lambda} f(a) /|f(a)|^{p}\right)+\log (1 / \lambda)} .
$$

Proof. I show this first for the fixed $b$ and then for $a \in \Omega$ by an automorphism. For any $\rho>0, S(b, \rho)$ is smoothly bounded and homeomorphic to a ball so there is function $h(\rho, \zeta)>0,0<\rho<r, \zeta \in \partial S(b, \rho)$ such that

$$
u(b) \leq \int_{\partial S(b, \rho)} h(\rho, \zeta) u(\zeta) d \sigma(\zeta)
$$

where $\sigma$ is surface area on $S(b, \rho)$ and $u$ is subharmonic. The function $h$ is smooth and bounded away from zero over all $\rho, \zeta$. Moreover $\int h(\rho, \zeta) d \sigma(\zeta)=1$. Averaging the inequality over $0<\rho<r$ yields

$$
u(b) \leq \int_{S(b, r)} u(z) H(z) d V(z)
$$

for some positive function $H$ which is bounded and bounded away from zero on $S(b, r)$, and $\int_{S(b, r)} H(z) d V(z)=1$. Apply this to $\log |f|$. This gives

$$
\log |f(b)|^{p} \leq \int_{S(b, r)} \log |f(z)|^{p} d m(z)
$$


where I have written $H(z) d V(z)=d m(z)$. Now, if we let $E:=E_{\lambda}(f, b)$ and $S:=S(b, r)$,

$$
\begin{aligned}
\log |f(b)|^{p} & =\int_{E} \log |f|^{p} d m+\int_{S \backslash E} \log |f|^{p} d m \\
& \leq m(E) \frac{1}{m(E)} \int_{E} \log |f|^{p} d m+\log \left(\lambda|f(b)|^{p}\right) m(S \backslash E), \\
\log |f(b)|^{p} & \leq m(E) \log \left[\frac{1}{m(E)} \int_{E}|f|^{p} d m\right]+\log \left(\lambda|f(b)|^{p}\right)(m(S \backslash E)), \\
0 & \leq m(E) \log \frac{A}{|f(b)| p}+\log \lambda(m(S \backslash E))
\end{aligned}
$$

where $A=(1 / m(E)) \int_{E} \log |f|^{p} d m$. Divide by $m(S)$ and solve for $m(S \backslash E) / m(S)$, remembering that $\log \lambda<0$. This gives

$$
\frac{m(S \backslash E)}{m(S)} \leq \frac{\log \left(A /|f(b)|^{p}\right)}{\log \left(A /|f(b)|^{p}\right)+\log (1 / \lambda)} .
$$

Since $H(z)$ is bounded above and below, $C m(S \backslash E) / m(S) \geq 1-$ $|E| /|S|$ and $A \leq C B_{\lambda} f(b)$; this gives (3). The result for arbitrary $a$ is obtained by observing that

$$
\frac{\left|S(a, r) \backslash E_{\lambda}(a)\right|}{|S(a, r)|} \sim \frac{\int_{S(a, r) \backslash E_{\lambda}(a)} B(z, z) d V(z)}{\int_{S(a, r)} B(z, z) d V(z)}
$$

where the symbol $\sim$ means the quantities have ratio bounded above and below, independent of $f$ and $a \in \Omega$. Now the right-hand side is equal to the same expression with $b$ replacing $a$ and $E_{\lambda}(f \circ Q, b)$ replacing $E_{\lambda}(a)$, where $Q$ is an automorphism taking $b$ to $a$. Similarly

$$
B_{\lambda} f(a) \sim B_{\lambda}(f \circ Q)(b) .
$$

So the general inequality follows from the one for $a=b$.

These lemmas are now put together exactly as in [5] to obtain the proof of the theorem. Choose the $\varepsilon$ in Lemma 2 so that $\varepsilon C<1 / 2$. Then, for this choice of $\varepsilon$.

$$
\int_{\Omega}|f|^{p} w d V \leq 2 \int_{\Omega \backslash B_{\varepsilon}}|f|^{p} w d V .
$$

If $a \in \Omega \backslash B_{\varepsilon}$, then $|f(a)|^{p}>\varepsilon B_{\lambda} f(a)$ provided $\lambda<1 / 2$. This inequality is improved by taking smaller $\lambda$, i.e. the choice of $\varepsilon$ is not 
dependent on $\lambda$. Apply (3) with this $a$ and $\varepsilon$ to get

$$
1-\frac{\left|E_{\lambda}(a)\right|}{|S(a, r)|} \leq C \frac{\log C \varepsilon^{-1}}{\log C \varepsilon^{-1}+\log (1 / \lambda)} .
$$

Now choose $\lambda$ small enough that the right-hand side of this inequality does not exceed $\delta / 2$. Since $|G \cap S(a, r)| /|S(a, r)|>\delta$ we must have $\left|E_{\lambda}(a) \cap G\right| /|S(a, r)|>\delta / 2$. This gives

$$
\frac{1}{\mid S(a, r)} \int_{G} X_{S(a, r)}(z)|f(z)|^{p} d V(z) \geq \frac{\delta}{2} \lambda|f(a)|^{p} .
$$

This will only be changed by a constant factor if we insert $w(z)$ on the left and $w(a)$ on the right. Integrate the result over $\Omega \backslash B_{\varepsilon}$ and use Fubini on the left to get

$$
\begin{array}{r}
C \int_{G}|f(z)|^{p} w(z)\left[\int_{\Omega \backslash B_{\varepsilon}} \frac{1}{|S(a, r)|} X_{S(a, r)}(z) d V(a)\right] d V(z) \\
\geq \frac{\delta}{2} \lambda \int_{\Omega \backslash B_{\varepsilon}}|f|^{p} w d V \geq \frac{\delta \lambda}{4} \int_{\Omega}|f|^{p} w d V .
\end{array}
$$

The argument used in Lemma 1 shows that the integral in brackets has a bound independent of $z$ and the result has been proved.

The converse of the theorem (i.e. that (1) implies there exists $r>0$ such that $L_{r}$ holds) requires some means of constructing a function in $A^{p}(w)$ which has appropriate behavior on $S(a, r)$. Suppose, for example, that $\Omega$ has finite volume and that $w(z)=B(z, z)^{\alpha}$ is integrable on $\Omega$, then $r>0$ can be chosen so that

$$
\int_{\Omega \backslash S(b, r)} w(z) d V(z)<\frac{1}{2 C} \int_{\Omega} w(z) d V(z) .
$$

Applying (1) to the function [det $\left.Q^{\prime}(z)\right]^{(2-2 \alpha) / p}$, where $Q$ is an automorphism of $\Omega$ taking $b$ to $a$, gives

$$
\begin{aligned}
\int_{G} B(z, z)^{\alpha}\left|\operatorname{det} Q^{\prime}(z)\right|^{2-2 \alpha} d V(z) & \\
& \geq \frac{1}{C} \int_{\Omega} B(z, z)^{\alpha}\left|\operatorname{det} Q^{\prime}(z)\right|^{2-2 \alpha} d V(z) .
\end{aligned}
$$


Changing variables $z \rightarrow Q(z)$ in (4) gives

$$
\begin{aligned}
\int_{\Omega \backslash S(a, r)} B(z, z)^{\alpha} \mid \operatorname{det} & \left.Q^{\prime}(z)\right|^{2-2 \alpha} d V(z) \\
\leq & \frac{1}{2 C} \int_{\Omega} B(z, z)^{\alpha}\left|\operatorname{det} Q^{\prime}(z)\right|^{2-2 \alpha} d V(z) .
\end{aligned}
$$

Combining (4), (5) and (6) gives

$$
\int_{G \cap S(a, r)} B(z, z)^{\alpha}\left|\operatorname{det} Q^{\prime}(z)\right|^{2-2 \alpha} d V(z) \geq \frac{1}{2 C} \int_{\Omega} w(z) d V(z) .
$$

The integral on the left is dominated by

$$
|G \cap S(q, r)| \sup \left\{B(z, z)^{\alpha}\left|\operatorname{det} Q^{\prime}(z)\right|^{2-2 \alpha}: z \in S(a, r)\right\} .
$$

Since $\left|\operatorname{det} Q^{\prime}(z)\right|^{2}=B(z, z) / B(Q(z), Q(z))$ we obtain

$$
C|G \cap S(a, r)| B(a, a) / B(b, b)^{1-\alpha}
$$

as a upper bound. Using $B(a, a) \sim 1 /|S(a, r)|$ gives, finally

$$
\frac{C|G \cap S(a, r)|}{|S(a, r)|} \geq \frac{1}{2 C} \int_{\Omega} w(z) d V(z) .
$$

This is the required inequality. Of course the validity of this argument depends on the function $\operatorname{det} Q^{\prime}(z)$ having a holomorphic $(2-2 \alpha) / p$ power. This will certainly be the case if $\Omega$ is simply connected or if $(2-2 \alpha) / p$ is an integer. Thus, for instance, the theorem and its converse hold in a ball or half-plane.

3. Let $F=\left\{z_{n}: n=1,2, \ldots\right\}$ be a sequence in $\Omega$ which is an $\eta$-net in the Bergman distance. That is, for every $z \in \Omega$ there is an integer $k$ with $d\left(z, z_{k}\right)<\eta$. It will also be assumed that $F$ is $\gamma$-separated for some $\gamma>0$, i.e. $d\left(z_{k}, z_{m}\right) \geq \gamma$ for all $k \neq m$. It is easy to see that for $f$ holomorphic in $\Omega$

$$
\left|f\left(z_{k}\right)\right|^{p} \leq \frac{C}{\left|S\left(z_{k}, \gamma / 2\right)\right|} \int_{S\left(z_{k}, \gamma / 2\right)}|f(z)|^{p} d V(z) .
$$

(Use the subharmonicity of $|f|^{p}$. Do the estimate at $z_{1}$ and transfer it to $z_{k}$ by an automorphism.) Moreover, the inequality (iii) of the theorem allows us to write

$$
\left|f\left(z_{k}\right)\right|^{p} \leq C B\left(z_{k}, z_{k}\right)^{1-\alpha} \int_{S\left(z_{k}, \gamma / 2\right)}|f(z)|^{p} B(z, z)^{\alpha} d V(z) .
$$


Since the $S\left(z_{k}, \gamma / 2\right)$ are disjoint,

$$
\sum_{k}\left|f\left(z_{k}\right)\right|^{p} B\left(z_{k}, z_{k}\right)^{\alpha-1} \leq \int_{\Omega}|f(z)|^{p} w(z) d V(z) .
$$

Thus, the restriction map $\left.f \rightarrow f\right|_{F}$ takes $A^{p}(w)$ into $L^{p}(F, \mu)$ where $\mu$ is the measure on $F$ that assigns to $z_{k}$ the mass $B\left(z_{k}, z_{k}\right)^{\alpha-1}$. (This can be found in the paper by Rochberg [6].)

There are two possibilities. Either the restriction map is one-to-one or it is not. In the former case, it will have closed range if and only if

$$
\int_{\Omega}|f|^{p} w d V \leq C \int_{F}|f|^{p} d \mu=C \sum_{k}\left|f\left(z_{k}\right)\right|^{p} B\left(z_{k}, z_{k}\right)^{\alpha-1}
$$

for some constant $C$. Referring to Lemma 1 , let $\varepsilon$ be chosen so that $\varepsilon C<1$ giving

$$
\int_{\Omega}|f|^{p} w d V \leq \frac{1}{1-\varepsilon C} \int_{\Omega \backslash A_{\varepsilon}}|f|^{p} w d V
$$

if $a \in \Omega \backslash A_{\varepsilon}$, then

$$
|f(a)|^{p}>\frac{\varepsilon}{|S(a, r)|} \int_{S(a, r)}|f|^{p} d V .
$$

As we saw in Lemma 2, this means there is a set containing $a$ with volume proportional to $\varepsilon^{2 n / p}$ times the volume of $S(a, r)$ in which $|f(z)|>$ $|f(a)| / 2$. Let $K_{a}$ denote this set. For $z \in K_{a}$

$$
|f(z)|^{p}>\frac{1}{2^{p}}|f(a)|^{p}>\frac{\varepsilon}{2^{p}} \frac{1}{|S(a, r)|} \int_{S(a, r)}|f(\zeta)|^{p} d V(\zeta) .
$$

Consequently,

$$
|f(z)|^{p} B(z, z)^{\alpha-1}>C \int_{S(a, r)}|f(\zeta)|^{p} B(\zeta, \zeta)^{\alpha} d V(\zeta) .
$$

In particular, if $M$ is the subset of those $k \in \mathbf{N}$, such that $z_{k}$ lies in $K_{a_{k}}$, some $a_{k} \in \Omega \backslash A_{\varepsilon}$, then

$$
\int_{S\left(a_{k}, r\right)}|f|^{p} w d V<C\left|f\left(z_{k}\right)\right|^{p} B\left(z_{k}, z_{k}\right)^{\alpha-1}, \quad k \in M .
$$

Sum over $M$ and define $G:=\cup_{k \in M} S\left(a_{k}, r\right)$.

$$
\int_{G}|f|^{p} w d V<C \sum\left|f\left(z_{k}\right)\right|^{p} B\left(z_{k}, z_{k}\right)^{\alpha-1}
$$


If $G$ satisfies the condition in the main theorem then (7) will be satisfied. Unfortunately the set $A_{\varepsilon}$ varies with $f$ and the above argument does not lead to a condition on $\left\{z_{k}\right\}$ alone. This can, however, be rectified.

THEOREM 2. Let $p>0, w(z)=B(z, z)^{\alpha}$ as before. Given $r>0$ and $\delta>0$ there exists a number $\beta>0$ with the following property: If $z_{k}$ is a separated sequence in $\Omega$ such that $G:=\cup S\left(z_{k}, \beta\right)$ satisfies $|G \cap S(a, r)|$ $>\delta|S(a, r)|$ for all $a \in \Omega$, then there is a constant $C>0$ such that

$$
\int_{\Omega}|f|^{p} w d V \leq C \sum\left|f\left(z_{k}\right)\right|^{p} B\left(z_{k}, z_{k}\right)^{\alpha-1} .
$$

REMARK. If $\left\{z_{k}\right\}$ is a $\beta$-net, then $G=\Omega$ and (8) is obtained. This follows from some arguments in [3]. However, $\left\{z_{k}\right\}$ need not be a $\beta$-net to obtain (8).

Proof. Let $C_{1}$ denote the constant in Theorem 1 for the present $G$ and let $C_{2}$ denote the constant in Lemma 1 . Choose $\varepsilon$ so that $\varepsilon C_{2}<1 / 2 C_{1}$. Then

$$
\int_{A_{\varepsilon}}|f|^{p} w d V \leq \frac{1}{2 C_{1}} \int_{\Omega}|f|^{p} w d V
$$

and

$$
\int_{G}|f|^{p} w d V>\frac{1}{C_{1}} \int_{\Omega}|f|^{p} w d V
$$

So

$$
\int_{G \backslash A_{\varepsilon}}|f|^{p} w d V>\frac{1}{2 C_{1}} \int_{\Omega}|f|^{p} w d V .
$$

The argument preceding the theorem can be applied. It has to be shown that each $a \in G \backslash A_{\varepsilon}$ satisfies $z_{k} \in K_{a}$ for some $k$. This will be the case if $\beta$ is chosen so that $S(a, \beta) \subseteq K_{a}$. To see that this can be done, note that $K_{a}$ is simply the image, under an automorphism which takes $S(b, r)$ to $S(a, r)$, of a fixed ball $K_{b}$ inside $S(b, r)$. Within $S(b, r), d(\cdot, \cdot)$ is equivalent to Euclidean distance, so $K_{b}$ contains $S(b, \beta)$ for sufficiently small $\beta$. If $Q$ is an automorphism of $\Omega$ taking $b$ to $a$, then $Q\left(K_{b}\right)=K_{a}$ and $Q(S(b, \beta))=S(a, \beta)$.

This shows that $\left\{z_{k}\right\}$ can be very far from being a $\beta$-net. The $\left\{z_{k}\right\}$ can occur in "clumps" which are arbitrarily far apart (i.e. $r>0$ is arbitrary) as long as the clumps have sufficiently many points. 
An argument similar to that in Theorem 2 also establishes the following. Let $\mu$ be any positive measure on $\Omega$.

THEOREM 3. If $A^{p}(w)$ is as in Theorem $2, r>0$, and $\delta>0$, then there exists $\beta>0$ with the following property: If there exist points $\left\{z_{k}\right\}$ satisfying the hypotheses of Theorem 2, and if there is a constant $\varepsilon>0$ such that $\mu\left(S\left(z_{k}, \beta\right)\right)>\varepsilon\left|S\left(z_{k}, \beta\right)\right|^{1-\alpha}$, then there exists $C>0$ such that $\int_{\Omega}|f|^{p} w d V$ $<C \int|f|^{p} d \mu$.

4. In $\S 3$, the case where the restriction is not one-to-one was not considered. It will be considered here in a one dimensional case, namely the unit disk. In this case the Bergman kernel gives $B(z, z)=\left(1-|z|^{2}\right)^{-2}$ and $A^{p}(w)$ is non-trivial for $w(z)=B(z, z)^{\alpha}$ only if $\alpha<1 / 2$. Define $B_{k}=B\left(z_{k}, z_{k}\right)^{\alpha-1}$ and let $l^{P}\left(\left\{B_{k}\right\}\right)$ denote the weighted sequence space $\left\{\left\{a_{n}\right\}: \sum\left|a_{n}\right|^{p} B_{n}<+\infty\right\}$. The sequences with only finitely many non-zero elements are dense in $l^{p}\left(\left\{B_{k}\right\}\right)$. If the restriction operator from $A^{p}(w)$ to $l^{p}\left(\left\{B_{k}\right\}\right)$ is not one-to-one then some non-zero $f \in A^{p}(w)$ must vanish on $\left\{z_{k}\right\}$. This implies that all finitely non-zero sequences belong to the range of the restriction operator. This gives half of the following

Proposition. Let $R$ denote the operator $R f=\left.f\right|_{\left\{z_{k}\right\}}$ defined on $A^{p}(w)$ (or on $H^{\infty}$ ). And suppose $R$ is not one-to-one. Then $R$ has closed range in $l^{p}\left(\left\{B_{k}\right\}\right)\left(\right.$ respectively, $\left.l^{\infty}\right)$ if and only if $R$ is onto.

Proof. In the $A^{p}(w)$ case the range contains a dense set. In the $H^{\infty}$ case the range contains the finitely non-zero sequences which are not dense. But standard Banach space arguments (the Open Mapping Theorem) show that there is a constant $M>0$ such that if $\left\{a_{k}\right\} \in$ range $R$, then there is an $f \in H^{\infty}$ with $\|f\|_{\infty} \leq M \cdot \sup _{k}\left|a_{k}\right|$ and $f\left(z_{k}\right)=a_{k}$. Now a normal families argument shows $R$ is onto.

In Rochberg's paper [6] a sufficient condition is obtained in order for $R$ to be onto in the $A^{p}(w)$ case. Roughly speaking, the condition is that $\left\{z_{k}\right\}$ be sufficiently separated in the Bergman metric. This gives the odd result that $R$ will have closed ranged if $\left\{z_{k}\right\}$ is either sufficiently separated or sufficiently dense!

In the case of $H^{\infty}$ where $R$ is one-to-one the results are considerably different. To have closed range, $R$ must satisfy $\|f\|_{\infty} \leq C\|R f\|_{\infty}$. Repeating this with $f^{n}$ in place of $f$ gives

$$
\|f\|_{\infty}^{n} \leq C\|R f\|_{\infty}^{n}
$$


Take $n$th roots and let $n \rightarrow \infty$ to get $\|f\|_{\infty} \leq\|R f\|_{\infty}$. Thus $\left\{z_{k}\right\}$ is a dominating set in the terminology of Rubel and Shields [7]. Brown, Shields and Zeller [2] showed $\left\{z_{k}\right\}$ is dominating if and only if almost every point of $|z|=1$ is a non-tangential limit of some subsequence of $\left\{z_{k}\right\}$. This gives the following solution for $H^{\infty}$.

Proposition. (Brown, Shields, Zeller.) If $\left\{z_{k}\right\}$ is a sequence in $|z|<1$ such that the restriction map on $H^{\infty}$ is one-to-one, then the restriction map has closed range if and only if almost every point of $|z|=1$ is a non-tangential limit point of $\left\{z_{k}\right\}$.

The last topic is the exhibition of a set $G$ in $\Omega=\{z \in \mathbf{C}=|z|<1\}$ which satisfies property $L_{r}$ for some $r$ and a function $f$ holomorphic in $\Omega$ which satisfies $\iint_{G}|f(z)|^{p} d x d y<+\infty$ but $\iint_{\Omega}|f(z)|^{p} d x d y=+\infty$. Let $G=\{z:|\arg (1+z) /(1-z)|>\pi / 4\}$. Then $G$ has two symmetric components, one in the upper half of $\Omega$, the other in the lower half. The upper component is a crescent shape bounded by two circular arcs. One such arc is the semi-circle of $|z|=1$ between -1 and +1 . The other is a circular arc with +1 and -1 as endpoints, passing through $(\sqrt{2}-1) i$. Then property $L_{r}$ is satisfied with $r>(\sqrt{2}-1)$.

On $G$ we have $\operatorname{Re}((1+z) /(1-z))^{2}<0$. Thus if

$$
f(z)=\exp ((1+z) /(1-z))^{2}
$$

then $f$ is bounded on $G$ but has an exponential singularity at $z=1$. So $\iint_{G}|f(z)|^{p} d x d y<+\infty$ but $\left.\iint_{\Omega} f(z)\right|^{p} d x d y=+\infty$.

5. Remarks. Lemmas 1-3 simplify considerably if the $S(a, r)$ are replaced by Euclidean balls $B(a, r(a))$ where $r(a)=\eta \operatorname{dist}(a, \partial \Omega)$ for some $0<\eta<1$. The theorem is true in that case but the result is not confromally invariant. At several points in Lemmas 1-3, I have used the fact that $B(z, z) d V(z)$ is invariant under automorphisms of $\Omega$. Similarly, it is invariant under biholomorphic transformations. That is, if $Q: \Omega_{1} \rightarrow \Omega_{2}$ is biholomorphic and $B_{i}$ is the Bergman kernel of $\Omega_{i}$; then

$$
\int_{\Omega_{2}} f(z) B_{2}(z, z) d V(z)=\int_{\Omega_{1}} f(Q(z)) B_{1}(z, z) d V(z) .
$$

Also, taking the case $\alpha=0, p=2$ for the moment

$$
f \rightarrow f(Q) \operatorname{det} Q^{\prime}
$$

is a unitary map of $A^{2}\left(\Omega_{2}\right)$ onto $A^{2}\left(\Omega_{1}\right)$. (Here $w \equiv 1$.) Now let $d \mu_{\imath}=$ $B_{i}(z, z) d V(z)$ on $\Omega_{i}$ and let $A^{2}\left(h_{i} d \mu_{i}\right)$ denote the space of analytic functions $f$ for which $\int_{\Omega_{l}}|f|^{2} h_{l} d \mu_{l}$ is finite. 
If $h_{i} \equiv B_{i}(z, z)^{-1}$ then $A^{2}\left(h_{i} d \mu_{i}\right)=A^{2}\left(\Omega_{i}\right)$. Let $Q: \Omega_{1} \rightarrow \Omega_{2}$ as before, then

$$
\int_{\Omega_{2}}|f(z)|^{2} B_{2}(z, z)^{-1} d \mu_{2}=\int_{\Omega_{1}}|f(Q(z))|^{2}\left|\operatorname{det} Q^{\prime}(z)\right|^{2} B_{1}(z, z)^{-1} d \mu_{1} .
$$

Let $\Omega_{1}$ be a domain satisfying the hypothesis of Theorem 1 and $G_{1}$ a subset satisfying $L_{r}$. Then $L_{r}$ may be reformulated as

$$
\mu_{1}\left(G_{1} \cap S(a, r)\right) \geq \delta \quad \text { for some } \delta>0 .
$$

Consequently, if $G_{2} \subseteq \Omega_{2}$ satisfies

$$
\mu_{2}\left(G_{2} \cap S(a, r)\right) \geq \delta
$$

then $G_{1}=Q^{-1}\left(G_{2}\right)$ satisfies the same inequality and so

$$
\begin{aligned}
\int_{G_{2}}|f(z)|^{2} B_{2}(z, z)^{-1} d \mu_{2} & =\int_{G_{1}}|f(Q(z))|^{2}\left|\operatorname{det} Q^{\prime}(z)\right|^{2} B_{1}(z, z)^{-1} d \mu_{1} \\
& \geq \frac{1}{C} \int_{\Omega_{1}}|f(Q(z))|^{2}\left|\operatorname{det} Q^{\prime}(z)\right|^{2} B_{1}(z, z)^{-1} d \mu_{1} \\
& =\frac{1}{C} \int_{\Omega_{2}}|f(z)|^{2} B(z, z)^{-1} d \mu_{2} .
\end{aligned}
$$

Thus, Theorem 1 is valid with (9) replacing $L_{r}$ in any domain biholomorphic to a domain satisfying the hypotheses of the theorem. In case $p \neq 2$ and $\alpha \neq 1$ the validity of this argument depends on the existence of certain analytic powers of $\operatorname{det} Q^{\prime}$. Thus, domains equivalent to a ball or a half-plane satisfy the Theorem (and its converse) with (9) replacing $L_{r}$.

If $\Omega$ is a product of domains for which the hypotheses (i)-(iii) hold then Theorem 1 holds in $\Omega$ provided $S(a, r)$ is replaced by the product of Bergman balls in the component domains. Thus, the result in [5] is valid in the polydisk.

Finally, one wonders how important the hypotheses on $\Omega$ are. In particular, does $\left(L_{r}\right) \Rightarrow(1)$ hold in, say, a smoothly bounded domain of holomorphy (non-homogeneous)? Also just when is hypothesis (iii) valid?

Added in proof. It has been pointed out to me that hypotheses (ii) and (iii) of Theorem 1 follow from (i) when $\Omega$ is bounded.

\section{REFERENCES}

[1] W. L. Bailey, Introductory Lectures on Automorphic Forms, Princeton University Press, 1973.

[2] L. Brown, A. L. Shields, and K. Zeller, On absolutely convergent exponential sums, Trans. Amer. Math. Soc., 96 (1960), 162-183. 
[3] R. R. Coifman and R. Rochberg, Representation theorems for holomorphic and harmonic functions, Asterisique, 77 (1980), 11-65.

[4] S. Kobayashi, Hyperbolic Manifolds and Holomorphic Mappings, M. Dekker, New York, 1970.

[5] D. H. Luecking, Inequalities in Bergman spaces, Illinois J. Math., 25 (1981), 1-11.

[6] R. Rochberg, Interpolation by functions in Bergman spaces, preprint.

[7] L. A. Rubel and A. L. Shields, The space of bounded analytic functions on a region, Ann. Inst. Fourier (Grenoble), 16 (1966), 235-277.

Received March 9, 1982. Supported in part by NSF Grant no. MCS-8201603.

UNIVERSITY OF ARKANSAS

FAYETTEVILLE, AR 72701 


\section{PACIFIC JOURNAL OF MATHEMATICS \\ EDITORS}

DONALD BABBITT (Managing Editor)

University of California

Los Angeles, CA 90024

Hugo RossI

University of Utah

Salt Lake City, UT 84112

C. C. MOOre and ARThur OGus

University of California

Berkeley, CA 94720
J. Dugundi

Department of Mathematics

University of Southern California

Los Angeles, CA 90089-1113

R. FINN and H. SAMELSON

Stanford University

Stanford, CA 94305

ASSOCIATE EDITORS

$\begin{array}{lllll}\text { R. Arens } & \text { E. F. Beckenbach } & \text { B. H. Neumann } & \text { F. Wolf } & \text { K. Yoshida }\end{array}$ $(1906-1982)$

\section{SUPPORTING INSTITUTIONS}

UNIVERSITY OF ARIZONA

UNIVERSITY OF BRITISH COLUMBIA

CALIFORNIA INSTITUTE OF TECHNOLOGY

UNIVERSITY OF CALIFORNIA

MONTANA STATE UNIVERSITY

UNIVERSITY OF NEVADA, RENO

NEW MEXICO STATE UNIVERSITY

OREGON STATE UNIVERSITY
UNIVERSITY OF OREGON

UNIVERSITY OF SOUTHERN CALIFORNIA

STANFORD UNIVERSITY

UNIVERSITY OF HAWAII

UNIVERSITY OF TOKYO

UNIVERSITY OF UTAH

WASHINGTON STATE UNIVERSITY

UNIVERSITY OF WASHINGTON 


\section{Pacific Journal of Mathematics}

\section{Vol. 110, No. $1 \quad$ September, 1984}

Wojciech Abramczuk, A class of surjective convolution operators $\ldots \ldots \ldots \ldots 1$

K. Adachi, Extending bounded holomorphic functions from certain

subvarieties of a weakly pseudoconvex domain $\ldots \ldots \ldots \ldots \ldots \ldots$

Malvina Florica Baica, An algorithm in a complex field and its application

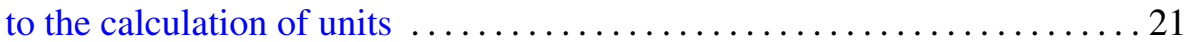

Giuliana Bianchi and Robert Cori, Colorings of hypermaps and a

conjecture of Brenner and Lyndon $\ldots \ldots \ldots \ldots \ldots \ldots \ldots \ldots \ldots \ldots \ldots \ldots$

Ronald James Evans, Determinations of Jacobsthal sums . . .......... 49

Leslie Foged, Characterizations of $\aleph$-spaces .................... 59

Nassif A. Ghoussoub and Paulette Saab, Weak compactness in spaces of

Bochner integrable functions and the Radon-Nikodým property . . . . . . 65

J. Gómez Gil, On local convexity of bounded weak topologies on Banach

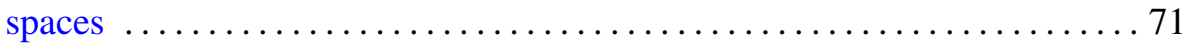

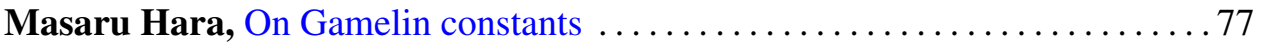

Wilfried Hauenschild, Eberhard Kaniuth and Ajay Kumar, Harmonic

analysis on central hypergroups and induced representations $\ldots \ldots \ldots 83$

Eugenio Hernandez, An interpolation theorem for analytic families of

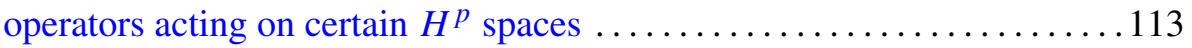

Thomas Alan Keagy, On "Tauberian theorems via block-dominated

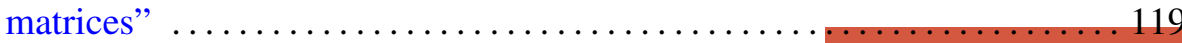

Thomas Landes, Permanence properties of normal structure $\ldots \ldots \ldots \ldots \ldots 125$

Daniel Henry Luecking, Closed ranged restriction operators on weighted

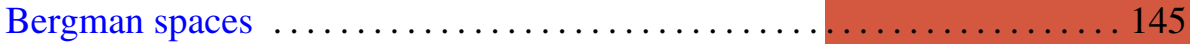

Albert Thomas Lundell, The $p$-equivalence of $\mathrm{SO}(2 n+1)$ and $\mathrm{Sp}(n) \ldots \ldots 161$

Mark D. Meyerson, Remarks on Fenn's "the table theorem" and Zaks' "the chair theorem" ..................................... 167

Marvin Victor Mielke, Homotopically trivial toposes . . . . . . . . . . 171

Gerard J. Murphy, Hyperinvariant subspaces and the topology on Lat A . . 183

Subhashis Nag, On the holomorphy of maps from a complex to a real manifold

Edgar Milan Palmer and Robert William Robinson, Enumeration of self-dual configurations ................................. 203

John J. Walsh and David Clifford Wilson, Continuous decompositions

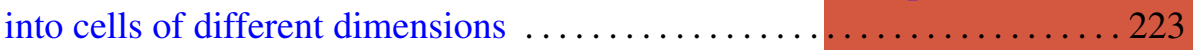

Walter John Whiteley, Infinitesimal motions of a bipartite framework .....233 